\title{
FREQUENCY OF ADENOMATOSIS COLI IN JAPAN ${ }^{1}$
}

\author{
Motoi Murata, ${ }^{2}$ Joji Utsunomiya, ${ }^{3}$ Takeo Iwama, ${ }^{3}$ \\ and Masako TANIMURA ${ }^{3}$ \\ ${ }^{2}$ Division of Genetics, National Institute of Radiological Sciences, \\ Chiba, 260 Japan \\ ${ }^{3}$ Second Department of Surgery and Polyposis Center, Tokyo Medical \\ and Dental University, Tokyo, 113 Japan
}

\begin{abstract}
Summary By using those cases of adenomatosis coli (AC) collected by Center for Polyposis Registration in Japan, an attempt was made for estimating frequency of the disease. Although year of diagnosis could be traced back to as early as 1954, the observed period was restricted to 1961 through 1975 because of their relatively constant performance for registration. All Japan was divided into prefectures and Tokyo Metropolitan Area alone was further subdivided into special wards $(k u)$. Cumulative incidence rate through all ages was proposed for an estimate of the frequency (f) of the disease at birth. Based on the observation that the secondary/ proband ratio was roughly 1.0 and on the assumption that those districts with largest values of incidence rate as the most likely estimator of the frequency, $f$ was estimated to be $5.74 \times 10^{-5}$. This estimate is agreeable with those obtained by other authors with different materials and methods.
\end{abstract}

\section{INTRODUCTION}

Adenomatosis coli $(\mathrm{AC})$ is one of the most common genetic diseases predisposing to human cancer. If a preventive colectomy would not be performed, the condition is ultimately fatal. Early detection of the condition or gene carriers will serve to prevent cancer development for years or through life. We have recently postulated that this disease is primarily responsible for a large proportion of the colo-rectal cancer in young adults of general population, e.g. younger than 40 (Utsunomiya et al., 1980). It is of great importance to evaluate the frequency of this disease or of the gene carriers among live borns in the general population. Several authors have already reported its estimate in various countries (Reed and Neel, 1955; Veale, 1965; Komatsu, 1968; Pierce, 1968; Alm and Licznerski, 1973); a crude estimate is con-

Received December 19, 1980

1 This work was partly supported by a Grant-in-Aid of Scientific Research Results, No. 501030 from the Ministry of Education, Science and Culture of Japan. 
ceived to be about one in ten thousands births. These authors utilized slightly different methods in data collection, and no one has yet tried to use incidence data derived from a nation-wide registry.

In Japan, the Center for Polyposis Registration has been operating at the Tokyo Medical and Dental University since 1976 and has been collecting family data for the cases of various types of familial polyposis syndrome reported from numerous hospitals in the whole country (Utsunomiya, 1977). It has also kept all the data gathered by the University for the last 20 years starting from the time of the first nation-wide survey for those diseases (Komatsu, 1968). While registration performance with regard to the rate of detecting cases may be as yet inadequate, it will be possible to evaluate tentatively the frequency of AC in Japan by using those collected cases.

\section{MATERIALS AND METHODS}

The Polyposis Center collected cases of various types of familial intestinal polyposis. Sources of data include random inquiries at hospitals, published case reports, autopsy records and voluntary referrals to the center by physicians. Diagnosis of the disease was pathologically confirmed in every case. For the definition of $\mathrm{AC}$, we took all the conditions with numerous adenomas (over 100) diffusely distributed throughout the colon and rectum, regardless of familial occurrence and associated extra-colonic changes. Thus, simple familial polyposis coli and Gardner's syndrome are included in it. The center collected 298 families of AC, by January of 1979, whose family pedigree was completed by using Koseki (an official household record) as well as a questionnaire to the hospitals. They comprise 298 probands and 214 secondary cases, of which most but about one fifth of the latter cases have been confirmed through either the clinical records of the hospitals or the death certificates. Although more than one probands were reported in each of about 30 families, the ascertainment was assumed to be single in the present study. Namely the first reported proband was chosen in every family for the proband group and the others were classified into the secondary cases. Sexes were not distinguished here. Table 1 shows the observed number of probands and secondary cases by years of diagnosis. In both groups number of reported cases varies from year to year. In the proband group it gradually increased from 1950's to 1965, reflecting the activity of the first survey study previously proceeded. After a short term reduction in 1966 and 1967, again it increased and reached maximum in 1972. The latter increase is owing to the activity of the present registry system. Falling off seen in most recent years may be due to a time lag between diagnosis and reporting. Performance of a central registry for a certain disease often experiences such a lag time of at most 4 or 5 years.

In the group of secondary cases the number increased also by years except in. most recent years. Unusually large numbers are recorded in 1972, 1974 and 1976 in comparison with that in the proband group. This excess probably corresponds to those cases who were diagnosed of polyposis after being called up by a clinician 
Table 1. Number of reported cases of AC patient by years of diagnosis.

\begin{tabular}{|c|c|c|c|c|}
\hline \multirow{2}{*}{ Year } & \multicolumn{2}{|c|}{ Probands } & \multicolumn{2}{|c|}{ Secondary cases } \\
\hline & Males & Females & Males & Females \\
\hline 1955 & 4 & 4 & 0 & 0 \\
\hline 1956 & 4 & 1 & 0 & 0 \\
\hline 1957 & 1 & 0 & 1 & 1 \\
\hline 1958 & 3 & 2 & 1 & 0 \\
\hline 1959 & 5 & 2 & 1 & 1 \\
\hline 1960 & 2 & 0 & 1 & 0 \\
\hline 1961 & 8 & 4 & 1 & 2 \\
\hline 1962 & 9 & 1 & 2 & 1 \\
\hline 1963 & 8 & 5 & 1 & 2 \\
\hline 1964 & 4 & 7 & 2 & 1 \\
\hline 1965 & 11 & 5 & 7 & 1 \\
\hline 1966 & 4 & 2 & 1 & 1 \\
\hline 1967 & 4 & 3 & 2 & 1 \\
\hline 1968 & 8 & 6 & 3 & 1 \\
\hline 1969 & 7 & 4 & 2 & 4 \\
\hline 1970 & 13 & 14 & 7 & 5 \\
\hline 1971 & 13 & 7 & 7 & 7 \\
\hline 1972 & 17 & 13 & 18 & 17 \\
\hline 1973 & 22 & 7 & 14 & 15 \\
\hline 1974 & 18 & 8 & 34 & 13 \\
\hline 1975 & 15 & 8 & 7 & 5 \\
\hline 1976 & 7 & 7 & 14 & 5 \\
\hline 1977 & 0 & 1 & 2 & 2 \\
\hline 1978 & 0 & 0 & 0 & 1 \\
\hline Total & 187 & 111 & 128 & 86 \\
\hline
\end{tabular}

for the purpose of examination before onset of any symptom. The call-up examination has gradually become practicable with the progress of family registration in recent years. Patients thus detected would, in a natural condition, visit a hospital at least a few years later. Inspecting the registry performance shown in Table 1, the observed period of the present study was defined as 15 years approximately, i.e. from 1961 to 1975 . Cases in the other years were added into the observed number. Nevertheless, estimation of incidence in individual districts should be fairly influenced by the fact that the registry performance in each year was quite variable by prefectures, and result in an underestimate.

Incidence rate of the disease (I) in the general population as well as frequency at birth (f) can be estimated in the following way. Incidence rate is given simply by dividing the number of cases (A) by the size of population $(\mathrm{N})$ as well as by the observed period (15 years). An arithmetic mean of the census populations in 1960 , 1965, 1970 and 1975 was used for the population size. Among 47 prefectures in Japan, Okinawa was excluded from the present study because it had not been affiliated with this registry system for almost entire period. Value of $A$, on the other 
hand, can be obtained by multiplying the number of probands (n) per year by the estimate of (proband + secondary)/proband ratio (a).

Several ways of estimating the frequency at birth for such a late onset disease as $\mathrm{AC}$ have been proposed. However no one seems exactly correct. This point will be discussed later. In the present study, a method of calculating a cumulative incidence rate over all ages is employed. It has become possible since the age at diagnosis is recorded for every year. Thus the frequency at birth is approximated by $f=a \cdot\left[1-\Pi_{t}\left(1-\frac{n_{t}}{15 \cdot N_{t}}\right)^{5}\right] \doteq \frac{a}{3} \cdot \sum_{t}\left(\frac{n_{t}}{N_{t}}\right)$ when age specific incidence rate, $\frac{\mathrm{n}_{\mathrm{t}}}{15 \mathrm{~N}_{\mathrm{t}}}$, is sufficiently small in any age class, t. Standard error of $\mathrm{f}$ is approximately given by $\frac{a}{3} \cdot \sqrt{\sum_{t} \frac{n_{t}}{N^{2}}}$.

Both I and f can be estimated for each of 46 prefectures by probands' residence. In this case $\mathrm{n}$ and $\mathrm{N}$ are both values for individual districts. Furthermore, Tokyo Metropolitan Area was divided into special wards $(k u)$ and other cities, since it has a tremendously large population size (about 10 percent of the whole country).

\section{RESULTS}

Table 2 gives the number of reported probands per $10^{6}$ person-years in individual prefectures. Census population in Japan excluding Okinawa was 93.4, 98.3, 103.1 and 110.2 millions in $1960,1965,1970$ and 1975 , respectively. Simple mean is 101.5 millions, while the number of probands is totally 298 . Thus the average values of $\mathrm{n} / \mathrm{N}$ in all Japan is $298 /(15 \times 101.5) \times 10^{6}$, or $0.196 / 10^{6}$. Variation among prefectures is of a considerable amount. It seems that this difference by regions is, though partly attributed to a chance effect, mainly caused by uneven activities in reporting as well as treating $\mathrm{AC}$ patients of the hospitals responsible for each district. Values for the upper five are 0.574 for Tottori, 0.476 for Mie, 0.418 for Aomori, 0.410 for Shimane and 0.374 for Shiga, respectively, per $10^{6}$. Several hospitals in these districts have been especially marked of their high activity with respect to the clinical investigation on this disease. On the other hand, values for those prefectures with lower most ranks are almost one tenth of the former five values. More conspicuous feature can be seen in Tokyo Metropolitan Area, within which a large amount of variation exists. When dividing the area into 23 special wards and other cities, there appeared several districts with a extremely large value, even much larger than Tottori, as is shown in Table 2. They are 0.936 for Toshima- $k u, 0.877$ for Minato-ku, 0.753 for Adachi- $k u$ and 0.625 for Suginami- $k u$, respectively, per $10^{6}$. The average for total special wards is 0.339 . These observations may indicate that the degree of success in detecting the disease by registry system is highly dependent on the number of active hospitals per unit population. In this context those values of upper ranks 
Table 2. Number of $\mathrm{AC}$ patients per $10^{6}$ person-years by prefectures. ${ }^{2}$

\begin{tabular}{|c|c|c|c|c|c|c|c|}
\hline \multirow{2}{*}{ Prefecture } & \multirow{2}{*}{$\begin{array}{l}\text { Census } \\
\text { population }\end{array}$} & \multicolumn{2}{|c|}{ No. of cases $/ 10^{6}$} & \multirow{2}{*}{ Prefecture } & \multirow{2}{*}{$\begin{array}{l}\text { Census } \\
\text { populationb }\end{array}$} & \multicolumn{2}{|c|}{ No. of cases $/ 10^{6}$} \\
\hline & & Proband & Secondary & & & Proband & Secondary \\
\hline Hokkaido & $5,182 \times 10^{3}$ & .116 & .051 & Aichí & $5,067 \times 10^{3}$ & .171 & .066 \\
\hline Aomori & 1,434 & .418 & .837 & Mie & 1,540 & .476 & .346 \\
\hline Iwate & 1,404 & .190 & .095 & Shiga & 891 & .374 & .225 \\
\hline Miyagi & 1,816 & .220 & .073 & Kyoto & 2,182 & .153 & .122 \\
\hline Akita & 1,272 & .157 & .000 & Osaka & 6,976 & .086 & .067 \\
\hline Yamagata & 1,257 & .106 & .159 & Hyogo & 4,425 & .105 & .045 \\
\hline Fukushima & 1,987 & .168 & .033 & Nara & 902 & .074 & .000 \\
\hline Ibaraki & 2,146 & .280 & .217 & Wakayama & 1,034 & .193 & .516 \\
\hline Tochigi & 1,578 & .211 & .042 & Tottori & 581 & .574 & .573 \\
\hline Gunma & 1,649 & .121 & .323 & Shimane & 813 & .410 & .082 \\
\hline Saitama & 3,531 & .245 & .283 & Okayama & 1,707 & .117 & .000 \\
\hline Chiba & 3,128 & .213 & .107 & Hiroshima & 2,383 & .168 & .168 \\
\hline Tokyo & 10,886 & .300 & .325 & Yamaguchi & 1,549 & .172 & .086 \\
\hline \multicolumn{2}{|c|}{ Area of wards 8,665} & .339 & .339 & Tokushima & 814 & .328 & .246 \\
\hline \multicolumn{2}{|c|}{ Other cities $\quad 2,221$} & .150 & .270 & Kagawa & 922 & .145 & .217 \\
\hline Kanagawa & 4,927 & .311 & .081 & Ehime & 1,457 & .092 & .000 \\
\hline Niigata & 2,397 & .083 & .028 & Kochi & 815 & .000 & .000 \\
\hline Toyama & 1,039 & .128 & .000 & Fukuoka & 4,067 & .295 & .099 \\
\hline Ishikawa & 1,005 & .133 & .000 & Saga & 872 & .153 & .000 \\
\hline Fukui & 754 & .177 & .177 & Nagasaki & 1,635 & .041 & .122 \\
\hline Yamanashi & 772 & .173 & .087 & Kumamoto & 1,760 & .038 & .000 \\
\hline Nagano & 1,977 & .337 & .169 & Oita & 1,192 & .056 & .000 \\
\hline Gifu & 1,739 & .230 & .039 & Miyazaki & 1,087 & .061 & .061 \\
\hline \multirow[t]{2}{*}{ Shizuoka } & 3,015 & .221 & .243 & Kagoshima & 1,817 & .073 & .000 \\
\hline & & & & Total & 101,497 & .196 & .141 \\
\hline
\end{tabular}

a Males and females are pooled. Prefecture map is presented in Fig. 1.

b Average of census populations in 1960, 1965, 1970 and 1975.

rather than the average for the whole country should be used in estimating I, unless any intrinsic variation in the gene frequency might be presumed.

Age structure of the population may influence the estimation of $I$ in each district, since this disease usually manifests itself in early and middle ages of life, i.e. prior to 50 years of age. So we tried to examine this effect. Since it was hard to get age specific incidence rates for every district because of meagerness of the collected cases, age adjustment was done by using the proportion of less than 50 years of age in population of each district. It varies from 0.69 to 0.87 among prefectures. Adjusted rate of $\mathrm{n} / 15 \mathrm{~N}$ thus obtained is, for instances, 0.630 for Tottori, 0.482 for Mie, 0.873 for Toshima- $k u$ and 0.818 for Minato- $k u$, respectively. Compared with the formerly presented values, increment or decrement is at most around 10 percent. Effect of age structure on the geographic variation seems to be minor. It was considered to be pertinent to use unadjusted values for further analysis.

In Table 2, number of secondary cases per $10^{6}$ person-years is also shown. The 


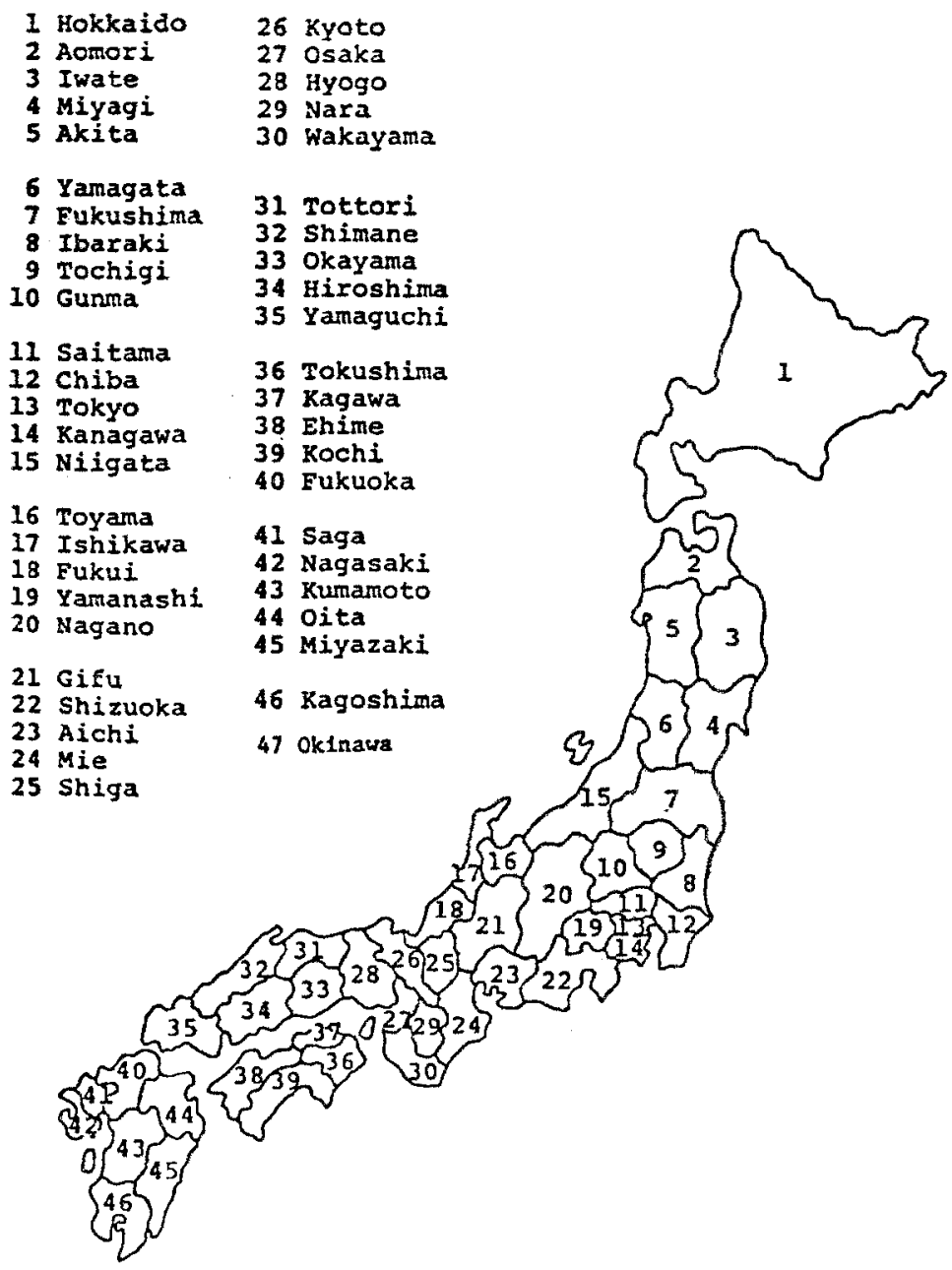

47

Fig. 1. Prefecture map of Japan.

order of magnitude is not necessarily compatible with that for the proband cases, but two out of three prefectures with upper most ranks are not changed. Mie is the fourth and Wakayama is now the third one. In Tokyo Metropolitan Area, value for Nakano- $k u$ and Edogawa- $k u$ are especially large. Mean value was about $0.141 / 10^{6}$. Comparing the observed number between proband and secondary cases, the secondary/proband ratio was calculated. It varied from 0.0 to 3.0 among prefectures. Using those data in Table 1 , the ratio was also calculated. Then it varied from 0.2 to 4.0 among different calendar years. Considering the facts that in the older years confirmation of the cause of death was more difficult than in recent years and that, to the contrary, in the recent years a certain part of secondary cases 
Table 3. Age specific incidence rate of AC in Japan (proband only).

\begin{tabular}{cccc}
\hline Age class & $\begin{array}{c}\text { Population } \\
\text { size }^{\mathrm{a}}\end{array}$ & $\begin{array}{c}\text { Number of } \\
\text { probands }^{\mathrm{b}}\end{array}$ & $\begin{array}{c}\text { Age specific } \\
\text { incidence rate }\end{array}$ \\
\hline $5-9$ & $8,528 \times 10^{3}$ & 4 & $.31 \times 10^{-7}$ \\
$10-14$ & 9,067 & 8 & .59 \\
$15-19$ & 9,266 & 17 & 1.22 \\
$20-24$ & 9,268 & 34 & 2.45 \\
$25-29$ & 9,120 & 48 & 3.51 \\
$30-34$ & 8,342 & 47 & 3.76 \\
$35-39$ & 7,533 & 45 & 3.98 \\
$40-44$ & 6,624 & 42 & 4.23 \\
$45-49$ & 5,733 & 31 & 3.61 \\
$50-54$ & 4,861 & 16 & 2.20 \\
$55-59$ & 4,173 & 14 & 2.24 \\
$60-64$ & 3,566 & 11 & 2.06 \\
$65-69$ & 2,792 & 4 & .96 \\
$70-74$ & 1,996 & 2 & .67 \\
$75-79$ & 1,237 & 1 & .54 \\
$80-$ & 900 & 0 & .00 \\
\hline Total & & 324 & \\
\hline
\end{tabular}

a Average of census populations in 1960, 1965, 1970 and 1975.

b Total number in the whole observed period of about 15 years.

was found by the call-up examination before onset of any symptom, 1.0 would be a reasonable approximation for the ratio. Then the estimate of a is 2.0 .

Utilizing those values of $\mathrm{n} / 15 \mathrm{~N}$ and a obtained above, $\mathrm{I}$ was estimated at $0.391 \times 10^{-6}$ for the mean of all Japan. For those districts with highest ranks, it was 1.146 in Tottori, 0.952 in Mie, 0.836 in Aomori, 0.820 in Shimane, 0.748 in Shiga and 0.677 in the special ward area of Tokyo, respectively, per $10^{6}$. If these six districts were chosen for the most likely estimator of $I$, notwithstanding the threshold for selection was very arbitrary, it has become $0.756 \times 10^{-6}$ with $\mathrm{n}$ and $\mathrm{N}$ being 79 and 13.9 millions, respectively.

Frequency of $\mathrm{AC}$ among live borns could be estimated as follows. In Table 3 the age distribution of diagnosis among AC probands $\left(\mathrm{n}_{t}\right)$ is presented. Note that the total number of cases is now 324, which differs from the previous one, i.e. 298. This is because a part of the former was classified into the secondary cases for estimating $I$, as was stated in the previous section. Number of individuals in the general population of each age class $\left(N_{t}\right)$ is also shown in Table 3 . With them the age specific incidence rate $\left(\frac{n_{t}}{15 N_{t}}\right)$ was calculated. Then, by summing the rates over all ages, the cumulative incidence rate was estimated to be $1.615 \times 10^{-5}$. Adjusting for the difference in total number of probands, i.e. between 298 and 324 , and further multiplying 2.0 for the parameter a, estimate of $f$ has become $(2.97 \pm$ $1.81) \times 10^{-5}$ for the mean of all Japan. However, as was mentioned formerly, selec- 
tion of six districts with upper most ranks is conceived more appropriate for the correct estimate of $f$. The estimate of $f$ in them was $(5.74 \pm 4.88) \times 10^{-5}$.

\section{DISCUSSION}

Several authors have already estimated the frequency of $\mathrm{AC}$ in different countries. Reed and Neel (1955) and Pierce (1968) obtained the frequency of 1.21 and 1.46 , respectively, per $10^{4}$ live borns in the United States, while Veale (1965) estimated it to be 0.42 in England. Their method utilized was almost the same. Survey for $\mathrm{AC}$ cases was depending on medical records of a specific hospital which was covering a resticted region almost completely. Method of statistical analysis is in brief presented by a formula, $f=P / D$, where $P$ stands for number of deaths with polyposis and $\mathrm{D}$ for total number of deaths in a certain district in a certain year. It assumes that the frequency at birth is practically the same with that in total deaths. Value of $\mathrm{D}$ can be obtained from vital statistics. For estimating $\mathrm{P}$, on the other hand, an indirect method had to be devised. They first estimated the number of deaths from large bowel cancer associated with polyposis before age 40 by multiplying the number of large bowel cancer deaths in the population with the proportion of $\mathrm{AC}$ cases among large bowel cancer patients in a university hospital. Then the age factor was adjusted by dividing the resultant with the proportion younger than 40 among those dying from large bowel cancer in AC cases collected separately for the purpose of a family study. The age of 40 was chosen because it was the mean age at death of $\mathrm{AC}$ patients. Accordingly their estimate of $f$ depends on three different sources, namely records of a specific hospital, data from a family study and vital statistics. It might introduce a considerable amount of error into the result as pointed out by Veale (1965). In addition, their assumption that the frequency at birth can be exemplified by that in deaths may not be correct. As stated by the authors, it assumes that no gene carrier will die from other causes than large bowel cancer. This should not be true. Furthermore this method may not be appropriate when the life expectancy in a general population has been significantly elongating in a relatively short period, because the patients with such a fatal condition should not be equally benefited with it.

Alm and Licznerski (1973) in Sweden, on the other hand, estimated the value of $1.31 \times 10^{-4}$ by using data of a national registry system. They declared that their estimate was more accurate than those of the previous authors, in that $\mathrm{P}$ as well as $\mathrm{D}$ could be obtained directly from the registry records. Nevertheless the question is still remained whether the frequency at death is well conforming to that at birth.

In Japan, too, Komatsu (1968) has already reported a frequency estimation with another-method. Data collection was done by a nation-wide survey of autopsy cases so that his estimate was as yet depending on the information on death. $\mathrm{He}$ investigated Annuals of the Pathological Autopsy Cases in Japan from 1958 to 1963 inclusive and found 8 cases out of 36,050 males and 4 cases out of 22,820 females 
being identifiable as AC. Since these autopsy cases have a different age distribution as well as a sex ratio from that of death cases in general presented in vital statistics, correction was made for them according to the latter as a standard. An adjusted number of $\mathrm{AC}$ was derived by multiplying the ratio of corrected/uncorrected number of deaths to the observed number of $\mathrm{AC}$ in individual age classes and thereafter summing over all age classes. Then $\mathrm{f}$ was estimated to be $1.34 \times 10^{-4}$ for males and $0.74 \times 10^{-4}$ for females. The author stated that the true value would be slightly smaller than the estimate because cases reported in above mentioned Annuals were usually restricted to those in major hospitals such as a university hospital or a national hospital.

In the present study an entirely different way of estimation was adopted. Cases were collected by a central registry system confined to the intestinal polyposis syndrome. As mentioned earlier, sources of data are owing to several different ways presently avilable. However, unlike the national registry in Sweden, the system does not receive a legal control. It absolutely depends on the physicians' voluntary report or on the activity of individual hospitals for this disease. The result in Table 1 and 2 obviously indicates that the registry performance widely varied with years as well as with districts. Therefore it should be clarified how completely we have collected the samples from the population survey. The segregation analysis may give an information regarding this point (Morton, 1959). However for such a genetic disease as $\mathrm{AC}$ which manifests its symptom in the adulthood stage, the method could not be applied in a usual way. We are now on a way of studying whole pedigrees comprehensively. An ascertainment probability was preliminarily estimated to be 0.26 from the distribution of probands among 306 affected sibships (Yasuda et al., in preparation). Penetrance rate could also be measured by way of a cumulative incidence rate among family members. Applying the life table method to totally 1,347 members ( 255 affected and 1,092 unaffected) excluding the probands and their parents among all families, the cumulative incidence rate was estimated to be slightly larger than 0.5 by age 60 . This means that penetrance should be almost complete when a bias due to other causes of death is excluded. Thus an ascertainment probability of 0.26 indicates that about one quarter of AC patients were detected as a proband on the average. Consequently the present estimate of f obtained for all Japan should be an underestimate, while that in the selected six districts would be a reasonable value.

With respect to the method of estimating $f$, a new statistical technique applying a cumulative incidence rate was utilized. The standard error was rather large compared with e.g. that of Reed and Neel (1955). This is because we assumed that the covariance term between age specific incidence rates of different ages was zero. Actually a negative correlation would be expected so that the standard error should be smaller than that presented here.

Besides the method developed by Reed and Neel (1955), other studies also have proposed different ways, though the subject of study was not AC. Reed and 
Chandler (1958) have estimated the frequency of Huntington's chorea by using the equation, $f=H / \sum_{t} N_{t} \cdot G_{t}$, where $H$ is the total number of prevalent cases observed at a certain time, $N_{t}$ is the total number of population of age $t$ and $G_{t}$ is the proportion of gene carriers whose chorea is recognized by age t. Note that their $\mathbf{H}$ differs from our incidence, A. Actually they call $f$ the frequency of heterozygotes in a population. But it must exactly correspond with the frequency at birth. $G_{t}$ is approximated from the observed age-at-onset curve by assuming a normal distribution. It was intended that the size of population in individual age classes should be weighted by their contribution to recognizing the disease by the time of observation. However it does not take into consideration the fatality of the disease, perhaps because the interval between onset and death is relatively long. On the other hand, Morton and Chung (1959), in studying the frequency of muscular dystrophy, have given a formula, $f=H / \sum_{t} N_{t} \cdot G_{t}\left[1-D_{t}\right]$, where $D_{t}$ is the cumulative proportion of death at age $t$ among affected subjects. In this case the factor of fatality is included.

It should be pointed out that the materials in the previous two studies are prevalence data, while they are incidence data in the present study. The latter can be converted into the former by multiplying the duration time or the interval from diagnosis to death of the disease. Namely the equation, $\mathrm{H}=\mathrm{A} \cdot \mathrm{y}$, holds good, where y stands for the duration time (years). Mean ages of diagnosis and death in our proband and secondary cases inclusive are 34.2 and 45.4 years, respectively, which provide 11.2 years for $y$. Ratio of $\left[\sum_{t} N_{t} \cdot G_{t}\left(1-D_{t}\right)\right] / N$ is also given from the age distributions of census population, diagnosis and death of affected cases. We have 0.205 . Thus the estimate of $f$ is now $0.756 \times 11.2 \times 10^{-6} / 0.205$ or $4.14 \times 10^{-5}$, which is slightly smaller than the former estimate. Anyhow it has become proven that both methods can provide more or less the same result in the frequency estimation of a late onset genetic disease. While ours is more laborious in attaining a satisfactory condition in data collection, it could give a more accurate result.

Mutation rate $(\mathrm{m})$ of the gene per generation can be estimated from $f$. By assuming that the population is in ballancing between mutational production and loss of the gene, $m$ is indirectly estimated from $m=f \cdot(1-W) / 2$, where $W$ is the relative fitness of the gene carrier (Haldane, 1935). Although presently the mutationselection ballance should be gradually relaxing by virtue of recent improvement in medical care for this disease, it might not as yet be an amount to affect the fitness of those cases studied. As an estimate of $W$, the relative reproductive span (Reed and Neel, 1955) can be provided from the present materials. It is approximately given by $R R S=\sum_{x} p_{x}\left(1-c \sum_{0}^{x} d_{i}\right)$, where $p_{x}$ is the fraction of deaths from polyposis in age class $\mathrm{i}$ among total affecteds and $\mathrm{c}$ is the probability for the gene carrier eventually die from polyposis. Now $\mathrm{c}$ is assumed to be unity, but it may not be true. Komatsu (1968) has demonstrated that the rate of infant death without diagnosis of polyposis 


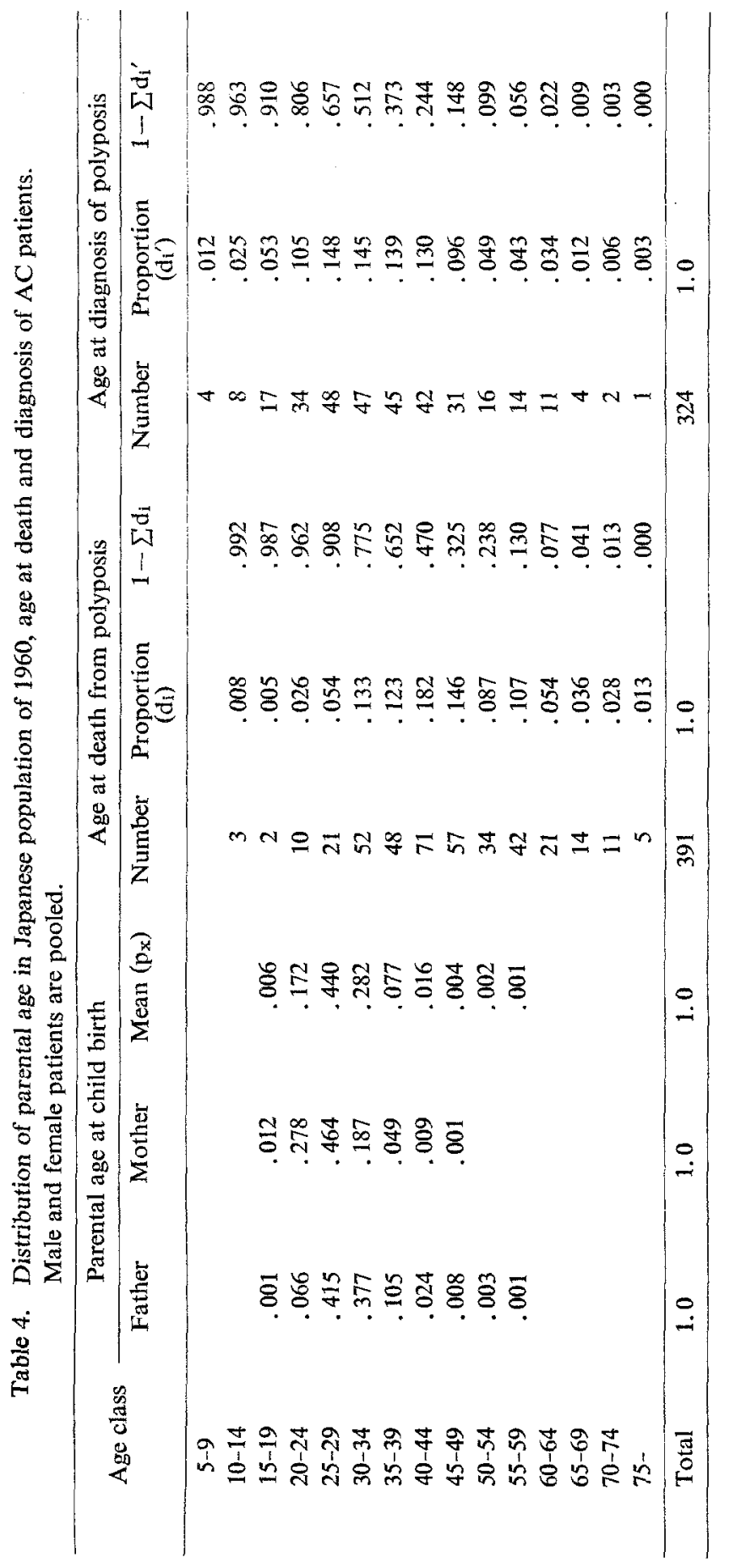


is considerably higher among female sibships of $\mathrm{AC}$ patients compared with that among control female population.

In Japan, parental age distribution at the time of child birth has been shifting in these decades, but it is not so extreme as to influence the result significantly. Hence for $p_{x}$, data from the vital statistics in 1960 were chosen and paternal and maternal age distributions were averaged (Table 4). Values for $d_{i}$ were calculated from the data presented in Table 3. It is conceivable, on the other hand, that AC patients may reduce their fertility substantially after the diagnosis and treatment. Therefore the proportion of diagnosis, $d_{i}{ }^{\prime}$, could be used in the place of $d_{i}$. RRS was estimated to be 0.85 or 0.61 , depending on whether $d_{i}$ or $d_{i}^{\prime}$ was adopted. As the present estimate of $\mathrm{W}$, we take the mean of them, 0.73 . Then $\mathrm{m}$ is $0.77 \times 10^{-5}$.

Acknowlèdgement The authors indebted to Dr. Norikazu Yasuda for kindly calculating the ascertainment probability in the present materials and also to Dr. Katsumi Tanaka for his careful reading and commenting upon the manuscript. They thank Miss Satsuki Hoshino for her technical help in preparing the manuscript.

\section{REFERENCES}

Alm, T., and Licznerski, G. 1973. The intestinal polyposis. Clin. Gastroenterol. 2: 577-602.

Haldane, J.B.S. 1935. The rate of spontaneous mutation of a human gene. J. Genet. 31: 317-326.

Komatsu, I. 1968. A clinical genetic study on multiple intestinal polyposis and allied conditions. Jap. J. Human Genet. 12: 246-297.

Morton, N.E. 1959. Genetic tests under incomplete ascertainment. Am. J. Hum. Genet. 11:1-16. Morton, N.E., and Chung, C.S. 1959. Formal genetics of muscular dystrophy. Am. J. Hum. Genet. 11: 360-379.

Pierce, E.R. 1968. Some genetic aspects of familial multiple polyposis of the colon in a kindred of 1,422 members. Dis. Colon \& Rect. 11: 321-329.

Reed, T.E., and Chandler, J.H. 1958. Huntington's chorea in Michigan. I. Demography and Genetics. Am. J. Hum. Genet, 10: 201-206.

Reed, T.E., and Neel, J.V. 1955. A genetic study of multiple polyposis of the colon (with an appendix deriving a method of estimating relative fitness). Am. J. Hum. Genet. 7: 236-263.

Utsunomiya, J. 1977. Present status of adenomatosis coli in Japan. In: Pathophysiology of Carcinogenesis in Digestive Organ (Farber, E. et al. eds.). University of Tokyo Press, Tokyo, pp. $305-321$.

Utsunomiya, J., Murata, M., and Tanimura, M. 1980. An analysis on the age distribution of colon cancer in adenomatosis coli. Cancer 45: 198-205.

Veale, A.M.O. 1965. Intestinal polyposis, Eugenics Laboratory Memoirs, XL., Cambridge University Press, London, pp. 104.

Vital Statistics 1975 Japan. Health and Welfare Statistics and Information Department, Ministry of Health and Welfare. 